\title{
Maximally Radio-Disjoint Multipath Routing for Wireless Multimedia Sensor Networks
}

\author{
Moufida Maimour CRAN laboratory \\ Nancy University, CNRS, France \\ Moufida.Maimour@cran.uhp-nancy.fr
}

\begin{abstract}
In wireless sensor networks, bandwidth is one of precious resources to multimedia applications. To get more bandwidth, multipath routing is one appropriate solution provided that inter-path interferences are minimized. In this paper, we address the problem of interfering paths in the context of wireless multimedia sensor networks and consider both intra-session as well as inter-session interferences. Our main objective is to provide necessary bandwidth to multimedia applications through non-interfering paths while increasing the network lifetime. To do so, we adopt an incremental approach where for a given session, only one path is built at once. Additional paths are built when required, typically in case of congestion or bandwidth shortage. Interference awareness and energy saving are achieved by switching a subset of sensor nodes in a passive state in which they do not take part in the routing process. Despite the routing overhead introduced by the incremental approach we adopt, our simulations show that this can be compensated by the overall achieved throughput and the amount of consumed energy per correctly received packet especially for relatively long sessions such as multimedia ones. This is mainly due to the fact that a small number of non-interfering paths allows for better performances than a large number of interfering ones.
\end{abstract}

\section{Categories and Subject Descriptors}

C.2 [Computer-Communication Networks]: Network Protocols

General Terms: Algorithms, Design, Performance

\section{INTRODUCTION}

Recent advances in micro-electronics and wireless communications in addition to the availability of inexpensive CMOS cameras and microphones, allowed for the emergence of wireless multimedia sensor networks (WMSN) 1]. These latters can significantly enhance a wide range of applications like object detection, surveillance, recognition, localization, and

Permission to make digital or hard copies of all or part of this work for personal or classroom use is granted without fee provided that copies are not made or distributed for profit or commercial advantage and that copies bear this notice and the full citation on the first page. To copy otherwise, to republish, to post on servers or to redistribute to lists, requires prior specific permission and/or a fee.

WMuNeP'08, October 27, 2008, Vancouver, BC, Canada.

Copyright 2008 ACM 978-1-60558-238-2/08/10 ...\$5.00. tracking. Such applications require a significant effort in developing new approaches well adapted to both wireless sensor networks and multimedia transport specific characteristics. On the one hand, sensor networks are very constrained in terms of energy, processing power and bandwidth. On the other hand, multimedia applications are resources hungry and are very demanding of bandwidth.

One solution to provide sufficient bandwidth to multimedia applications in WSN and hence improve their quality, is multipath routing that has been a hot research area for years. Most of multipath routing protocols in wireless sensor [4] 6] and generally in ad hoc networks [11] [9] were targeted to failure tolerance. Additional paths are maintained to serve as backup on primary path failure. Few of them like SMR [8] consider the use of multiple paths concurrently. In SMR, two or more maximally disjoint routes are used simultaneously. Disjointness [10] 13] allows for a more balanced traffic in the network however, it does not deal with interferences. The selected paths may be highly interfering if their respective links are too close. Consequently, the overall achieved throughput is far from being the summation of the bandwidth offered independently by the different paths [15.

Because of the shared nature of the medium and the non centralized random access protocols in ad hoc wireless network, interferences are likely to appear when two active (transmitting/receiving) nodes are in the radio range of each other. One approach to reduce interferences is the design of routing protocols with new metrics that integrate interferences in their paths cost. Some of these metrics are ETX (the expected transmission count metric) [2] and ETT (the expected transmission time metric) 3]. Extensions of these metrics have recently been proposed considering the availability of a multi-channel facility in mesh networks [3] [12. More recently 14 proposed WIM (Weighted Interference Multipath metric) that allows for considering spatial diversity through introducing the number of neighbors in the estimation of interferences.

All the previously cited works on interference-aware routing, rely on metric estimations that require frequent periodic probing messages exchange. This is not suitable to WSN characterized by their scarce energy and limited processing capabilities. In fact, most of these metrics are targeted to mesh networks where these limitations do not hold. Moreover, they adopt a source-routing approach which is, once again, not suitable to WSN because of RREQ packets size requiring more energy to be transmitted/received. Finally, proposals relying on a multi-channel facility are bet- 
ter suitable to mesh networks while they are not adapted to WSN due to the overhead and energy consumption needed for channel switching.

In this paper, we consider the problem of interfering paths in WMSN. We assume that only one channel is available and thus we do not address intra-path interferences. Instead, we focus on inter-path interferences and consider both intrasession (for one source) and inter-session (typically, multiple sources) interferences. Our main objective is to provide necessary bandwidth to multimedia applications through noninterfering (radio disjoint [7]) paths while increasing the network lifetime. To achieve our twofold goal, we chose to adopt an incremental approach where only one path is built at once for a given session. Additional paths are built when required, typically in case of path congestion or lack of bandwidth. When a given path is selected to be used, all nodes interfering with it are put in a passive state. Passive nodes do not further take part in the routing process so they could not be used to form a new path that consequently, will not interfere with previously built ones. Moreover, passive nodes can be put in sleep or idle modes, thus allowing for energy saving and hence increasing the network lifetime.

Despite the routing overhead introduced by the incremental approach we adopted, our simulations showed that this can be compensated by the overall achieved throughput and the amount of consumed energy per correctly received packet especially for relatively long sessions such as multimedia ones compared to traditional scalar data ones. This is mainly due to the fact that a small number of non-interfering paths allows for better performances than a large number of interfering ones. The paper is organized as follows. Our incremental non-interfering multipath protocol is described in section 2 Some simulation results are shown in section 3. Section 4 concludes and summarizes some of our future work.

\section{MAXIMALLY RADIO-DISJOINT MULTIPATH ROUTING (MR2)}

Our proposal is an incremental on-demand reactive multipath routing protocol that makes use of path tables at the sensor nodes. Each sensor is able to create, maintain and update a path table that records the different paths to the sink. It contains an entry for each path with the following fields :

- pathId, the path id, corresponds to the last crossed sensor in this path from the source to the sink.

- nextNode, the next hop toward the sink on this path,

- metric, an estimation of the associated quality metric for this path (hop count, available energy, an estimation of path lifetime or any other metric depending on the application requirements).

- inUse, a flag when set, it indicates that the corresponding path is currently in use.

A sensor node with respect to the routing process can be either in active or passive state. In a passive state, as opposed to an active one, a sensor does not take part in the routing process. From an energy point of view, a passive node can be put in an idle or even a sleep mode to save energy and hence increasing the network lifetime. This depends on the network density and if concurrent sessions with critical information have to be handled. Clearly, a tradeoff is to be made between energy saving and serving other sessions mainly critical ones. We argue that putting some nodes in a sleep mode is well justified in the case of dense networks as it is the case of WSN.

\subsection{Route discovery}

In order to build paths, MR2 follows main ideas behind existing routing algorithms in ad-hoc and sensor networks. The sink floods the network with a request until the sensor, referred to as the source, having the requested data is reached. A request contains the following fields:

- a request sequence number that gives the rank of the currently built path for this session,

- a path id that corresponds to the first crossed sensor from the sink by this request,

- the last crossed node id,

- till this node path quality metric

- a flag called isRepair set when a path has to be repaired (the currently built path is to replace a broken one)

Initially, all sensor nodes are in the active state and route discovery is initiated by the sink sending a request using its address as the path $i d$. Upon the reception of a request, a sensor node creates a new path entry if the reported path id does not appear in its table. If the request originator is the sink, then the path $\_i d$ of the entry is this node id ; otherwise, it uses the path id reported by the current request. The nextNode field is simply the last crossed sensor by the request and the inUse flag is set to zero. The metric field is also updated depending on its nature. If for instance, we choose to use hop count as our metric, then the reported metric is incremented by one before it is recorded. If the reported path $i d$ is already stored in the path table, then the corresponding entry is replaced if the current request provides a better quality metric ; otherwise, the request is simply ignored. A request needs to be rebroadcast only if it induces path table update.

Every time, the source receives a request, it records it in its path table. When all routes from all of its active neighbors are received, the source proceeds for the selection of one path with the best metric value. Immediately, it begins transmitting data on this chosen path. When a sensor node receives a data packet to be forwarded on a given path $P$, it sets the inUse flag and sends (only once per session) a bePassive message to its neighbors excepting its next and previous nodes in $P$. On the reception of a bePassive, a node changes its state to passive. When an additional path has to be built, passive nodes do not react to requests and hence will not take part in the formation of an additional path. In this way, we ensure a maximum radio disjointness among built paths, thus improving the application overall throughput.

As described, MR2 is designed in the context of a requestdriven sensor network model where the sink is the originator of a request. However, it can be easily extended to the event-driven model where a sensor detecting a target decide to transmit sensed data to the sink. In fact, paths can be 
built using the flooding of the first data packet transmitted by the source toward the source for instance.

\subsection{Data transmission}

An application data packet header mainly contains a sequence number, the identity of the source in addition to the path id on which this packet has to be forwarded. Depending on the application, the construction of an additional path can be initiated by the sink immediately after receiving the first data packet or when a given condition occurs. In the case of video transport for instance, the sink may require building an additional path when the video quality is poor. An additional path discovery can also be requested when the sink detects congestion when it experiences larger inter-packet delays than expected.

When multiple paths are available, the source has to be able to partition its traffic on the available paths following a given strategy depending on the application nature and requirements. If reliability is of a prime importance, one can choose to transmit redundantly the same flow on more than one path. In contrary, when the application is semi-reliable where some losses are tolerated as it is the case of multimedia and in particular image/video delivery, the traffic can be divided into multiple flows using layered or multiple description coding. In the former, there is one base layer and several enhancement layers and in the latter, flows are of equal importance.

\subsection{Route maintenance}

Route maintenance is the role of the sink. A broken path is detected by the sink if it does not receive further data on this path for a given period of time or when it receives a RERR (route Error) message. This latter is sent by a sensor when its energy level is under a given threshold. When a path is reputed to be broken, the sink initiates a new route discovery phase with the isRepair field set. The request, in this case, contains an additional field that records the id of the broken path. In this way, the source is able to know which entry it has to remove from its path table.

\section{SIMULATION RESULTS}

Our interference-aware routing protocol (MR2) is implemented using TOSSIM, a bit level simulator for TinyOS platform. We also implemented the single path approach in addition to a multipath routing protocol without interference awareness. In all of the implemented schemes, the quality metric used is path length given as the number of hops from the source to the sink. In what follows, we will use terms Single and $H C$ to refer to the single path approach and the multipath scheme without interference awareness.

In our simulations, we considered a square sensor field of size $1000 \times 1000 \mathrm{~m}^{2}$ where a given number $N^{2}$ of static sensor nodes ranging from 49 to 625 are deployed in a randomized grid. The sink is located at the upper right corner (coordinates 1000,1000) and a given number of transmitting sources are selected randomly. Experiments were performed and averaged over 100 simulations with different topologies and two different densities corresponding to what we refer to as sparse and dense modes. In the former, the transmission radio range for all nodes is set to $1500 / N$ and in the latter to $2000 / N$ giving a mean node degree of 8 and 12 respectively.

All the sensors have same processing capability and the energy dissipation due to processing was neglected in our

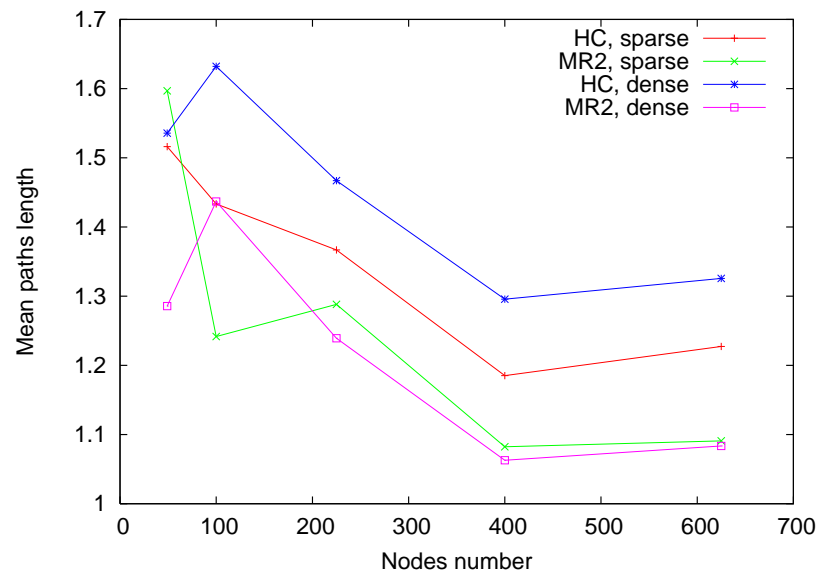

Figure 1: Paths length ratio with respect to Single

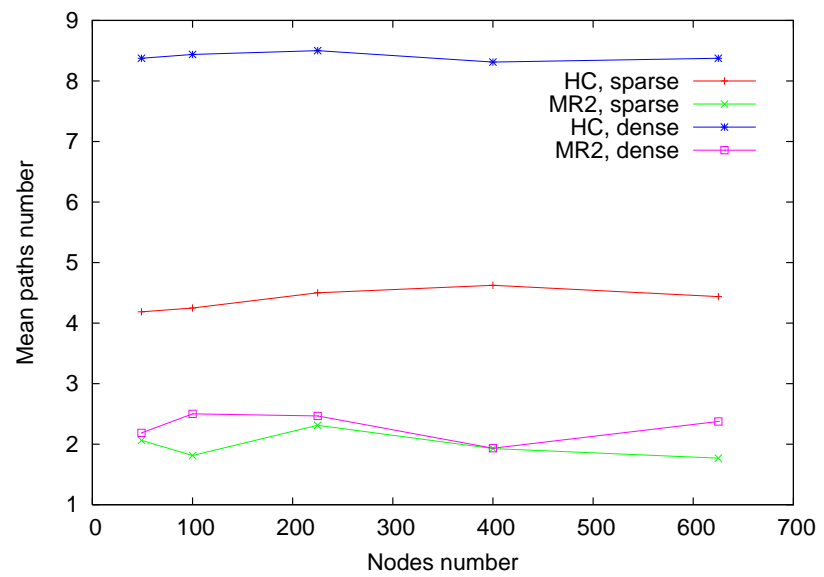

Figure 2: Number of built paths

simulations. For communication, we adopted the energy model of $[5]$. To transmit a k-bit message a distance $\mathrm{d}$, the consumed energy is given by $E_{T x}(k, d)=E_{\text {elec }} k+\epsilon_{a m p} k d^{2}$. To receive a k-bit message, a sensor consumes $E_{R x}(k)=$ $E_{\text {elec }} k$ where $E_{\text {elec }}$ is the dissipated energy by the radio to run the transmitter or the receiver circuitry and $\epsilon_{a m p}$ is the required energy by the transmit amplifier. The passive nodes are put in the sleep state and assumed to consume $1 / 100$ the reception power.

Firstly, we looked at the characteristics of built paths in the different implemented schemes. Figure 1 plots the mean path length (hops number) ratio of built paths in MR2 and $\mathrm{HC}$ with respect to the case of a single path scheme. One fundamental observation is that MR2 paths are closer to the best path length (single path scheme) especially when increasing the network size/density. Figure 2 gives the mean number of built paths as a function of the number of nodes. We see that in MR2, we are able to only build a restricted number of paths (about 2, due to interference-awareness) compared to $\mathrm{HC}$ where more than 4 or 8 paths are built depending on the network density. Despite that, MR2 achieves better performances in terms of overall achieved throughput, energy saving and end-to-end delay even with a few number of paths as will be shown in what follows. 


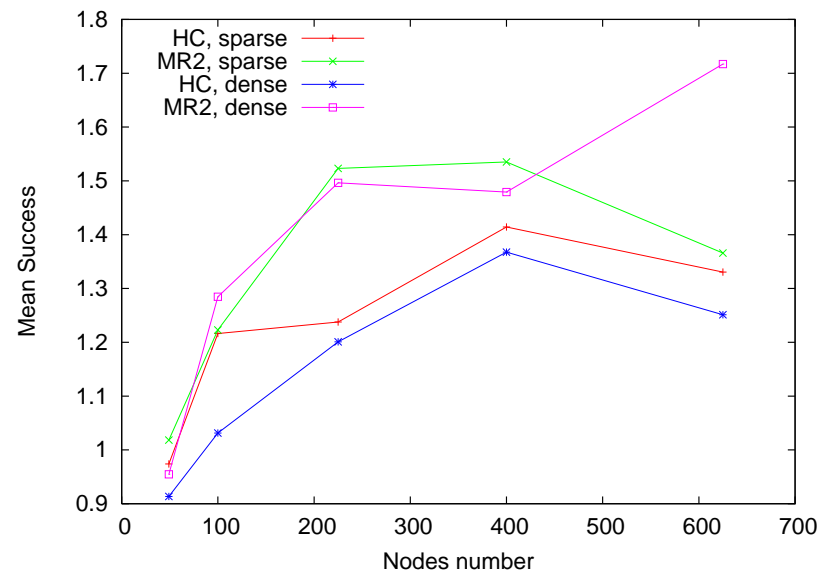

Figure 3: Success ratio with respect to a single path approach

Table 1: Number of involved nodes in the delivery process

\begin{tabular}{|c||c|c|c||c|c|c|}
\hline \multicolumn{1}{|c||}{ Mode } & \multicolumn{3}{c||}{ Sparse } & \multicolumn{3}{c|}{ Dense } \\
\hline Scheme & HC & MR2 & ratio & HC & MR2 & ratio \\
\hline 49 nodes & 24.54 & 12.75 & 0.52 & 24.54 & 9.81 & 0.4 \\
\hline 100 nodes & 45.69 & 16.85 & 0.37 & 45.69 & 13.75 & 0.3 \\
\hline 225 nodes & 88.02 & 42.6 & 0.48 & 88.02 & 32.33 & 0.37 \\
\hline 400 nodes & 130.02 & 49.35 & 0.38 & 130.2 & 35.13 & 0.27 \\
\hline 625 nodes & 164.49 & 58.41 & 0.36 & 164.40 & 55.81 & 0.34 \\
\hline
\end{tabular}

Figure 3 plots the overall success ratio of the two multipath approaches with respect to the single path delivery strategy as a function of the number of nodes. We can see that MR2 achieves better success ratio that increases with the network size especially for highly interfering networks (dense mode). For instance, MR2 improves the success ratio by more than $70 \%$ (with only two paths) compared to a single path approach instead of only $30 \%$ for $\mathrm{HC}$ (with more than 8 paths). Figure 4 shows the mean throughput improvement for the different sources as a function of time and confirms the previous observations. Mainly, we observe that in the dense mode, MR2 clearly shows better performances. However, in the sparse mode, achieved throughput is almost the same. Despite that, MR2 still performs better in terms of resources utilization (number of involved sensors in the delivery process) due to its restricted number of paths. Table 1 reports the number of involved nodes in the routing process in the two multipath schemes in both sparse and dense modes for different network sizes. We can see that in MR2, a small number of nodes contribute to achieve at least the same level of performances as HC.

Achieved improvements in MR2 are the result of the absence of interferences thanks to our incremental approach in building paths. Figure 5 shows how much each built path contributes in the delivery success. We see that one path in MR2 gets more than $50 \%$ of success which allows for achieving more throughput with only two paths than the single path approach. However the contribution of one path when

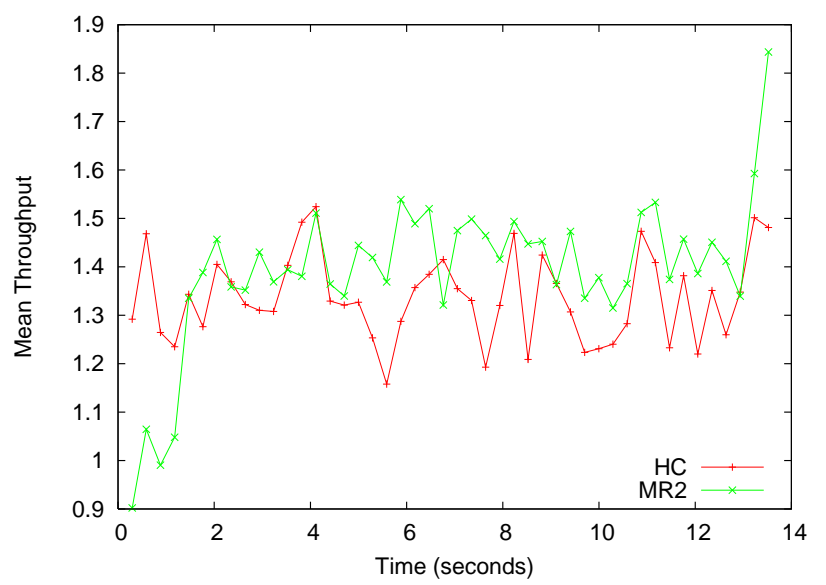

(a)

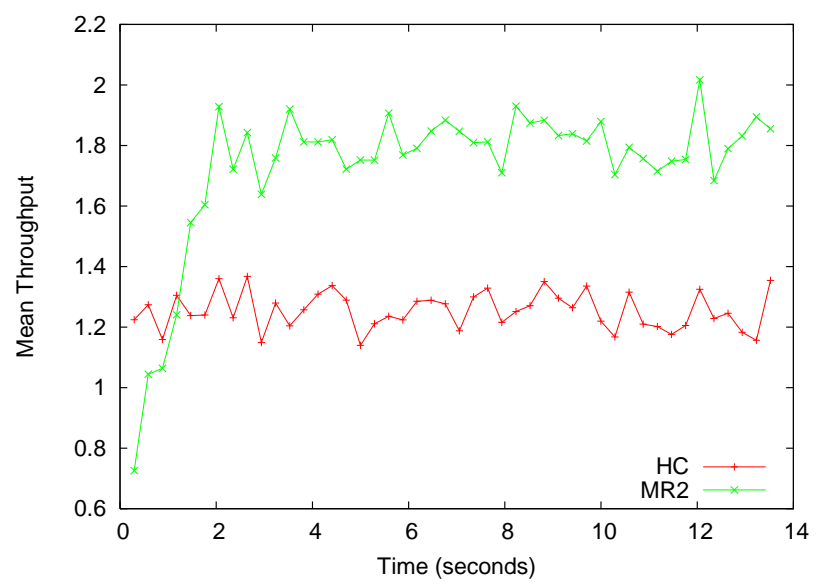

(b)

Figure 4: Achieved throughput with respect to a single path approach for 625 nodes (a) sparse mode, (b) dense mode

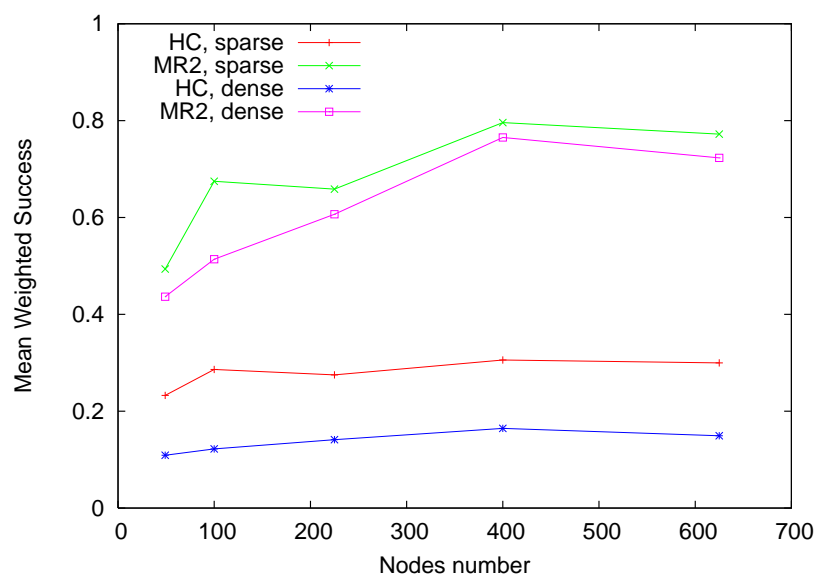

Figure 5: Success ratio per built path with respect to a single path approach. 


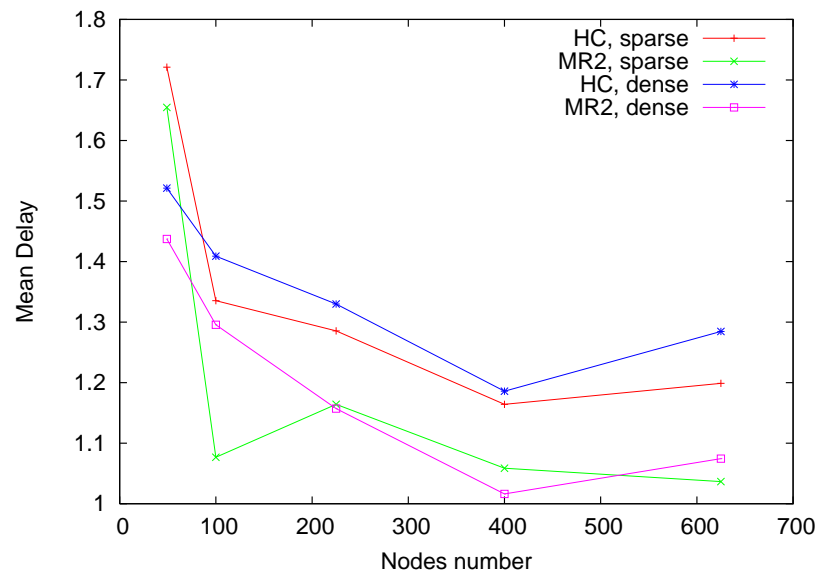

Figure 6: Delay with respect to a single path approach

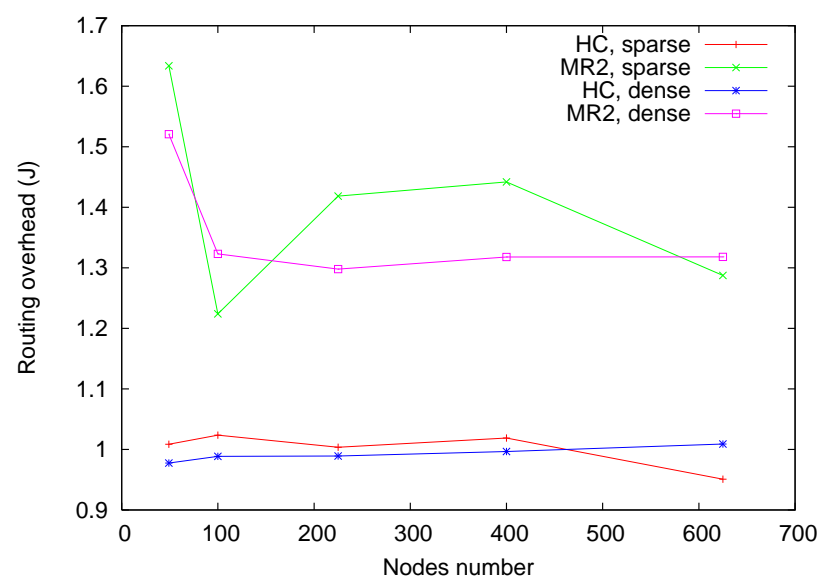

Figure 7: Overall routing overhead

interferences are frequent (HC) is about $20 \%$. This observation shows how it is important to build non-interfering paths.

In terms of the end-to-end delay, figure 6 shows that once again, MR2 performs better since paths length is smaller than those built by the non-interference aware approach (HC). As already shown (figure 1), MR2 paths length are closer to the smallest path built in the single path routing. This behavior is more noticeable when the number of nodes increases. This is a nice feature for scalability and to avoid big variations in delay between different paths serving the same session.

In order to evaluate the routing overhead introduced by the incremental approach adopted in MR2, we performed simulations for one session per source of 15 seconds and others for 5 minutes to see the energy saving that we can achieve when a session delay increases. We evaluate routing overhead using the consumed energy by mainly the request messages. Figures 7 and 8 plot energy consumption of the routing process as a function of the network size with respect to single path routing. As expected, MR2 as shown in figure (7) introduces about $30 \%$ of additional overhead compared to HC. However, when we look to the experienced overhead per

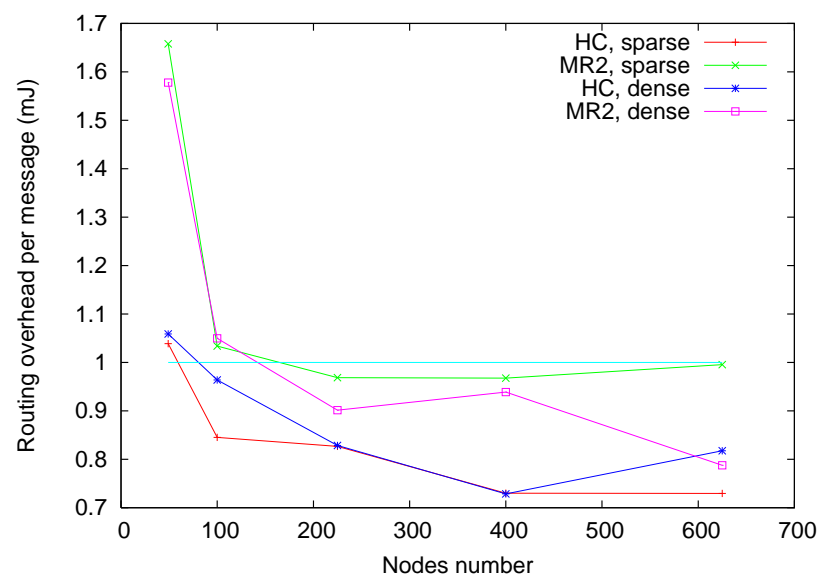

Figure 8: Per message routing overhead

correctly received packet (figure 8), we see that the difference is less pronounced even if $\mathrm{HC}$ introduces less routing overhead.

Let us now look to the overall energy consumption per message including routing overhead. Figure 9 shows this amount of energy for the case of short sessions (15 seconds) and long ones ( 5 minutes). We can see that for both cases, MR2 consumes less energy especially for highly interfering and large networks. It is clear that energy saving will be better if we increase the simulation time as shown in figure 9(b). This is mainly due to the fact that a given number of nodes (passive ones) are put in the sleep mode.

We finally, looked at the load fairness among the sensors involved in the delivery process in terms of the number of processed messages. We used $\left(\sum_{i=1}^{N_{a}} M_{i}\right)^{2} /\left(N_{a} \sum_{i=1}^{N_{a}} M_{i}^{2}\right)$ where $M_{i}$ is the number of processed messages by node $i$ and $N_{a}$ is the number of the involved nodes in delivering data. Figure 10 shows that a better distribution of energy consumption is achieved in MR2. This allows for avoiding nodes to be more loaded than others. This decreases the probability that a given node consumes all of its energy before other ones and consequently, the network lifetime will be improved.

\section{CONCLUSION}

In this paper, we addressed the problem of interfering paths in the context of WMSN and considered both intrasession as well as inter-session interferences. We adopted an incremental approach where for a given session, only one path is built at once and additional paths are built when required. Interference awareness and energy saving are achieved through putting some nodes in a passive state. Simulation results showed that despite the routing overhead introduced by the incremental approach we adopted, this was compensated by the overall achieved throughput and the amount of consumed energy per correctly received packet especially for relatively long sessions such as multimedia ones. This is mainly, as shown, due to the fact that a small number of non-interfering paths allows for better performances than a large number of interfering ones. As a future work, we expect to implement both ETX and ETT metrics in order to compare our approach to those based on these two metrics. One application to congestion control and image transport 


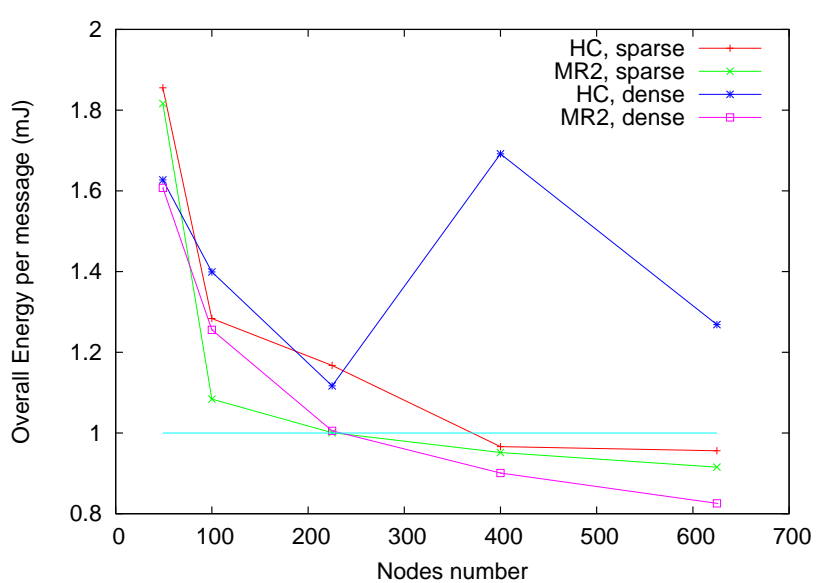

(a)

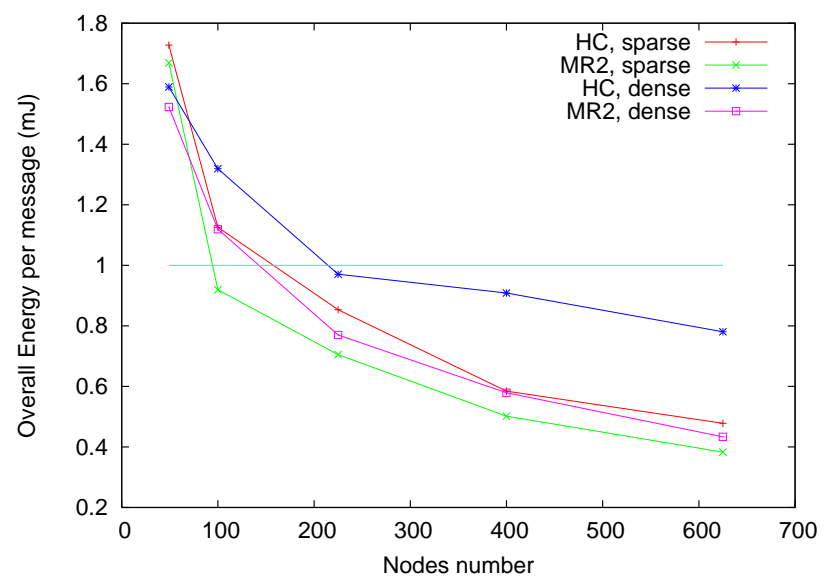

(b)

Figure 9: Overall consumed Energy per message (including routing overhead) in a session of (a) 15 seconds, (b) 5 minutes

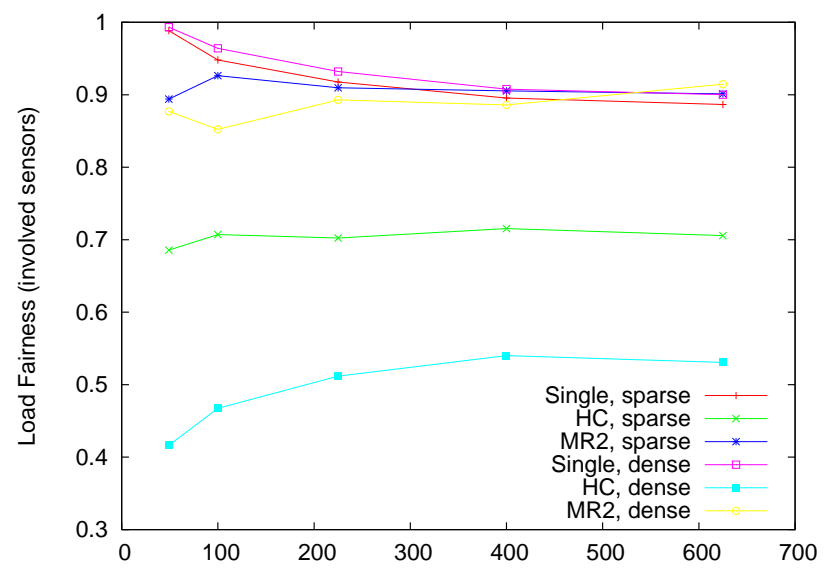

Figure 10: Load fairness among involved sensors is under development. We also plan to perform experiments in a real WSN test-bed where our approach will be applied to image/video transport in order to get more insight into the real benefit we could obtain.

\section{ACKNOWLEDGMENTS}

This work was supported by French National Research Agenecy (ANR TCAP project, No. 06-JCJC-0072).

\section{REFERENCES}

[1] I. F. Akyildiz, T. Melodia, and K. R. Chowdhury. A survey on wireless multimedia sensor networks. Computer Networks, 51(4):921-960, March 2007.

[2] D. S. J. De Couto, D. Aguayo, J. Bicket, and R. Morris. A high-throughput path metric for multi-hop wireless routing. In Proceedings of the 9th ACM International Conference on Mobile Computing and Networking (MobiCom '03), San Diego, California, September 2003.

[3] R. Draves, J. Padhye, and B. Zill. Routing in multi-radio, multi-hop wireless mesh networks. In MobiCom '04: Proceedings of the 10th annual international conference on Mobile computing and networking, pages 114-128, New York, NY, USA, 2004. ACM.

[4] D. Ganesan, R. Govindan, S. Shenker, and D. Estrin. Highly-resilient, energy-efficient multipath routing in wireless sensor networks. ACM SIGMOBILE Mobile Computing and Communications Review, 5(4):11-25, 2001.

[5] W. Heinzelman, A. Chandrakasan, and H. Balakrishnan. Energy-efficient communication protocol for wireless microsensor networks. In Proceedings of the 33rd Hawaii International Conference on System Sciences (HICSS'OO), January 2000.

[6] X. Hou, D. Tipper, and J. Kabara. Label-based multipath routing (lmr) in wireless sensor routing. In Proceedings of the 6th International Symposium on Advanced Radio Technologies (ISART 04), Boulder, CO, March 2-4 2004.

[7] K. Kuladinithi, M. Becker, C. Görg, and S. Das. Radio disjoint multi-path routing in manet. In In CEWIT (Center of Excellence in Wireless and Information Technology), pages $1-2,2005$.

[8] S. Lee and M. Gerla. Split multipath routing with maximally disjoint paths in ad hoc networks. In IEEE ICC, volume 10, pages 3201-3205, 2001

[9] S. J. Lee and M. Gerla. Aodv-br: Backup routing in ad hoc networks. In Proceedings of the IEEE Wireless

Communications and Networking Conference (WCNC 2000), Chicago, IL, Sepember 2000.

[10] Y. Lu and V. W. Wong. An energy-efficient multipath routing protocol for wireless sensor networks. Wiley International Journal of Communication Systems, special issue on Energy-efficient Networks Protocols and Algorithms for Wireless Sensor Networks, 2006.

[11] A. Nasipuri and S. Das. On-demand multipath routing for mobile ad hoc networks. pages 64-70, 1999.

[12] I. Sheriff and E. M. Belding-Royer. Multipath selection in multi-radio mesh networks. In BROADNETS. IEEE, 2006.

[13] A. Srinivas and E. Modiano. Minimum energy disjoint path routing in wireless ad-hoc networks. In Proceedings of the ACM/IEEE International Conference on Mobile Computing and Networking (MOBICOM), September 2003.

[14] J. Tsai and T. Moors. Interference-aware multipath selection for reliable routing in wireless mesh networks. In MeshTech. IEEE, 2007.

[15] S. Waharte and R. Boutaba. Totally disjoint multipath routing in multihop wireless networks. In Proceedings of the IEEE International Conference on Communications (ICC 2006), Istanbul, Turkey, June 2006 